\title{
Ultrasoft Colloid-Polymer Mixtures: Structure and Phase Diagram
}

\author{
B. Lonetti, ${ }^{1}$ M. Camargo, ${ }^{2,3,4}$ J. Stellbrink, ${ }^{1}$ C. N. Likos, ${ }^{4}$ E. Zaccarelli, ${ }^{5}$ L. Willner, ${ }^{1}$ P. Lindner, ${ }^{6}$ and D. Richter ${ }^{1}$ \\ ${ }^{1}$ JCNS-1 and ICS-1, Forschungszentrum Jülich, Leo-Brandt-Straße, 52425 Jülich, Germany \\ ${ }^{2}$ Institute of Theoretical Physics, Heinrich-Heine Universität Düsseldorf, D-40225 Düsseldorf, Germany \\ ${ }^{3}$ Dirección Nacional de Investigaciones, Universidad Antonio Narino, Kra 3 Este 47a-15 Bogotá, Colombia \\ ${ }^{4}$ Faculty of Physics, University of Vienna, Boltzmanngasse 5, A-1090 Vienna, Austria \\ ${ }^{5}$ CNR-ISC and Dipartimento di Fisica, Università di Roma La Sapienza Piazzale A. Moro 2, I-00185, Roma, Italy \\ ${ }^{6}$ Institute Laue-Langevin, 6, rue Jules Horowitz, 38042 Grenoble CEDEX 9, France
}

(Received 28 February 2011; published 31 May 2011)

\begin{abstract}
Binary mixtures of ultrasoft colloids and linear polymer chains were investigated by small-angle neutron scattering and liquid state theory. We show that experimental data can be described by employing recently developed effective interactions between the colloid and the polymer chains, in which both components are modeled as point particles in a coarse-grained approach, in which the monomers have been traced out. Quantitative, parameter-free agreement between experiment and theory for the pair correlations, the phase behavior and the concentration dependence of the interaction length is achieved.
\end{abstract}

DOI: 10.1103/PhysRevLett.106.228301

Hard spheres have been established in the past as model systems to investigate on a fundamental level the effective interactions and phase behavior of soft matter [1]. Following in complexity, colloid-polymer mixtures are usually described by the Asakura-Oosawa (AO) model [2]. Building on these simple models, great advances have been made in the study of gel and glass formation in colloidal systems [3,4]. More recently, the interest of colloid scientists has shifted towards the study of soft particles, among which star polymers have emerged as a model for a wide class of soft spheres, including blockcopolymer micelles [5,6] and microgel particles [7,8]. For a star polymer, softness can be controlled by varying its number of arms (or functionality $f$ ), allowing to bridge the gap between linear polymer chains $(f=2)$ and hard spheres $(f \rightarrow \infty)$ [9]. Therefore, star polymers feature tunable softness, which is responsible for the observation of anomalous structural behavior [9] and for the formation of several crystal structures [5,10]. Mixtures of soft particles offer a much higher versatility with respect to their hard counterparts, both in terms of structural and rheological properties [11-13] and of effective interactions [14]. In particular, mixtures of star polymers of different sizes and functionalities have been recently investigated in a joint theoretical and experimental effort, revealing the existence of multiple glassy states [15]. On the other hand, the paradigmatic case of a mixture of star polymers and linear chains (the direct soft counterpart of the AO model) has been investigated theoretically $[14,16]$ and experimentally by (mainly) macroscopic rheology $[11,12,17]$; however, detailed structural information is still missing. Recently a microscopic theory $[14,16]$, capable to appropriately coarse-grain stars and chains, has been developed, but an accurate comparison between theoretical predictions and
PACS numbers: 82.70.-y, 61.20.Gy, 64.70.km, 82.70.Dd

experimental results for the structural correlations for starchains mixtures has not been attempted so far.

Recently, we introduced kinetically frozen starlike micelles $[6,18]$ formed by the amphiphilic block copolymer poly(ethylene-alt-propylene)-poly(ethylene oxide), PEPPEO, as a tunable model system for ultrasoft colloids [9]. In this Letter we study mixtures of starlike micelles and linear (PEO) chains and provide a systematic and quantitative characterization of structure factors and phase behavior in terms of effective interactions. By combining small-angle neutron scattering (SANS) and liquid state theory, we measure and model the correlations between starlike micelles and linear chains. SANS measurements in core contrast allow a direct determination of experimental structure factors, $S(Q)$ [19], providing the basis for a comparison with the recently-developed theory, in which both components are modeled as point particles in a coarse-grained approach that traces out the monomeric degrees of freedom. A direct comparison without any adjustable parameters, i.e., using the quantities directly given by experiments, provides a very good agreement between experiment and theory for structure factors, phase behavior and concentration dependence of the interaction length $\sigma$. The comparison is done for a broad range of polymer volume fraction from dilute to concentrated conditions, i.e. $0.05 \leq \phi / \phi^{*} \leq 7$, where $\phi^{*}$ is the overlap concentration, and it brings forward the influence of the added chains on the larger star polymers. Our work provides a comprehensive structural characterization of soft binary mixtures in terms of effective potentials and is a further step forward in the understanding and modelling of complex soft matter systems in general.

Partially deuterated PEP-PEO block polymers and partially deuterated PEO chains were synthesized by anionic 
polymerization following established procedures [20]. Molecular details of the systems are given in Table I. All polymers were dispersed in a $\mathrm{H}_{2} \mathrm{O} / \mathrm{D}_{2} \mathrm{O}$ mixture (5:95, $v: v$ ) to ensure a PEO contrast matching (core contrast). SANS experiments were performed at D11, Institute LaueLangevin (ILL), Grenoble. Raw data were corrected for detector efficiency and dead time. Contributions resulting from empty cell, solvent and incoherent scattering were subtracted and corrected data finally normalized by a water standard to absolute units $\left[\mathrm{cm}^{-1}\right]$.

Characterization of the single starlike micelle was performed in dilute solution. The SANS-form factor $P(Q)$ was analyzed using a core-shell model, by assuming a starlike density profile in the shell [6]. Light scattering (SLS/DLS) and rheology were used as complementary methods. A compilation of experimental data gives a functionality $f=91 \pm 5$ and the characteristic sizes shown in Table I. The gyration radii yield a chain-micelle size ratio $\xi=$ $R_{g}^{(c)} / R_{g}^{(s)} \cong 0.3$, which was fixed for the subsequent theoretical analysis.

A detailed description of our theoretical approach is given in $[14,16]$. By taking the center of the stars and the middle monomer of the chains as effective coordinates, the coarse-grained entropic pair interactions display a logarithmic dependence at short distances and an exponentially decay at large ones. Since the potentials diverge only logarithmically as $r \rightarrow 0$, both star and chains can be viewed as ultrasoft colloidal particles [9], which can easily interpenetrate and overlap with one another. The star-star interaction reads as [9]

$$
\beta V_{s s}(r)=\Theta(f)\left\{\begin{array}{ll}
-\ln \left(\frac{r}{\sigma_{s}}\right)+\frac{1}{1+\frac{\sqrt{f}}{2}} & r \leq \sigma_{s} \\
\frac{\sigma_{s} / r}{1+\frac{\sqrt{f}}{2}} \exp \left[-\frac{\sqrt{f}}{2 \sigma_{s}}\left(r-\sigma_{s}\right)\right] & r>\sigma_{s}
\end{array},\right.
$$

where $\Theta(f)=\frac{5}{18} f^{3 / 2}, f$ is the functionality, $\sigma_{s}$ is a measure of the star extension and $\beta=\left(k_{B} T\right)^{-1}$ with $k_{B}$ the Boltzmann constant and $T$ the temperature. The chains can be seen as two-arm stars interacting via [21,22]

$$
\beta V_{c c}(r)=\Theta(2) \begin{cases}-\ln \left(\frac{r}{\sigma_{c}}\right)+\frac{1}{2 \tau^{2} \sigma_{c}^{2}} & r \leq \sigma_{c} \\ \frac{1}{2 \tau^{2} \sigma_{c}^{2}} \exp \left[-\tau^{2}\left(r^{2}-\sigma_{c}^{2}\right)\right] & r>\sigma_{c}\end{cases}
$$

TABLE I. Characterization of the used polymers.

\begin{tabular}{lcccc}
\hline \hline \multicolumn{5}{c}{ Molecular characteristics $\left(\left[M_{w}\right]=\mathrm{g} / \mathrm{mol}\right)$} \\
Sample & $M_{w, \text { PEP }}$ & $M_{w, \text { PEO }}$ & $M_{w} / M_{n}{ }^{\mathrm{a}}$ & $x_{D}{ }^{\mathrm{b}}$ \\
\hline hPEP1-dhPEO20 & 1100 & 21250 & 1.03 & 0.86 \\
dhPEO20 & $\cdots$ & 21250 & 1.03 & 0.86 \\
Characteristic sizes $(\AA)$ & & & \\
$R_{g}^{(s)}$ & $R_{\text {core }}^{(s)}$ & $R_{\text {shell }}^{(s)}$ & $R_{h}^{(s)}$ & $R_{g}^{(c)}$ \\
$218 \pm 11$ & $34 \pm 2$ & $248 \pm 12$ & $317 \pm 16^{\mathrm{c}}$ & $67 \pm 3$ \\
\hline \hline
\end{tabular}

${ }^{\text {a}}$ Polydispersity by SEC.

${ }^{\mathrm{b}}$ Molar fraction of deuterated PEO.

${ }^{\mathrm{c}}$ Hydrodynamic radius obtained from DLS. with $\tau \sigma_{c}=1.03$, which guarantees the correct value of the second virial coefficient of a polymer solution. As before, $\sigma_{c}$ measures the size of the chain. The star-chain interaction results [16]

$$
\beta V_{s c}(r)= \begin{cases}-\hat{\Theta}(f) \ln \left(\frac{r}{\sigma_{s c}}\right)+K & r \leq \sigma_{s c}, \\ v_{0} \int \varrho_{s}\left(r^{\prime}\right) \varrho_{c}\left(\left|\mathbf{r}-\mathbf{r}^{\prime}\right|\right) d \mathbf{r}^{\prime} & r>\sigma_{s c}\end{cases}
$$

where $\sigma_{s c}=\frac{1}{2}\left(\sigma_{s}+\sigma_{c}\right), \quad \hat{\Theta}(f)=\frac{5}{36} \frac{1}{\sqrt{2}-1}\left[(f+2)^{3 / 2}-\right.$ $\left.\left(f^{3 / 2}+2^{3 / 2}\right)\right]$, and $v_{0}$ is the excluded volume parameter. The latter comes from Flory-type arguments for the overlapping monomer density profiles $\varrho_{i}(r)(i=s, c)$, which can be evaluated on the basis of the Daoud-Cotton blob model [16,23,24]. The constants $K$ and $v_{0}$ are estimated by requiring continuity of $V_{s c}(r)$ and $V_{s c}^{\prime}(r)$ at $r=\sigma_{s c}$. In Fig. 1 the effective potentials $V_{s s}, V_{s c}$ and $V_{c c}$ are shown, from which center-to-center structure factor of stars $S(Q)$ follows by solving the two-component Ornstein-Zernike (OZ) equations within the hypernetted-chain (HNC) approximation. The latter is very accurate for soft-core repulsive potentials $[25,26]$.

For our quantitative comparison between experiments and theory the following quantities are necessary: the reduced number densities of starlike micelles, $\rho_{s} \sigma_{s}^{3}$, and of linear chains, $\rho_{c} \sigma_{s}^{3}$, unambiguously given by experimental concentrations in terms of volume fraction, as well as $f$ and $\xi$, both obtained from independent form factor analysis. Following [16,21], the relation $\sigma_{i} \simeq 4 R_{g}^{(i)} / 3$, $i=s, c$ holds. According to the theoretical predictions, upon the addition of linear chains, a strong loss of star-star correlations results, insofar a drastic decrease of the peaks of $S(Q)$ is found in comparison to the structure factor of the pure star system. This change is accompanied by a shift of the peaks to slightly higher $Q$ and also by a slight increase of the $S(Q \rightarrow 0)$ value. These features can be well explained based on a depletionlike mechanics as described in [11]. The inset in Fig. 2 demonstrates the strong weakening of the peak of the starlike micelle scattering intensity upon increasing the chain density at fixed star density $\rho_{s} \sigma_{s}^{3}=0.367$. For direct comparison, theoretical $S(Q)$ were multiplied by $P(Q)$ and convoluted with the instrumental resolution function [27].

Figure 2 shows SANS intensities $I(Q)$ for selected concentrations below and around the overlap polymer volume

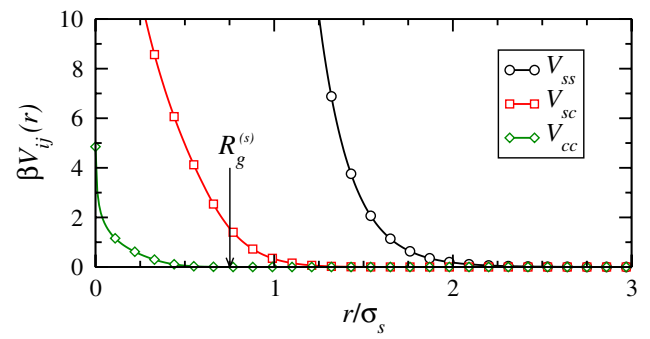

FIG. 1 (color online). Star-star, star-chain, and chain-chain pair effective potentials given by Eqs. (1)-(3) for $\xi=0.3$. 


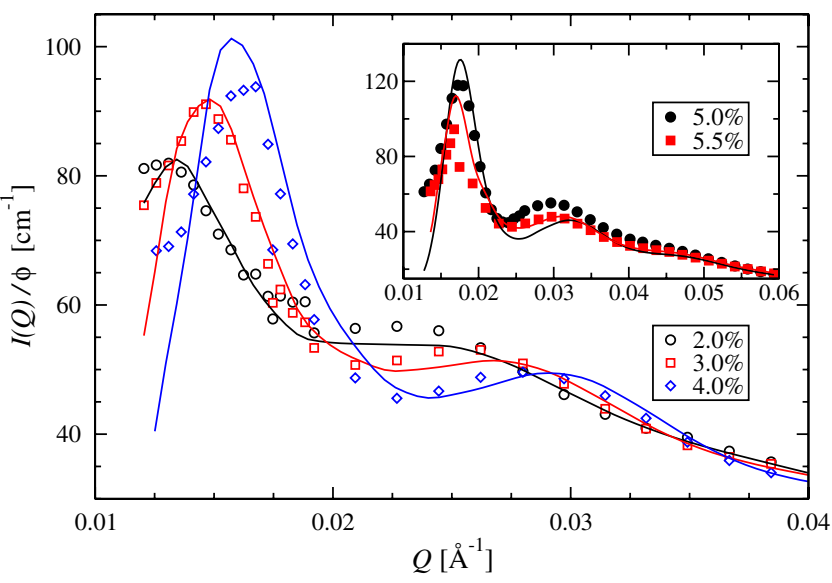

FIG. 2 (color online). Normalized SANS intensity for intermediate polymer volume fractions. Inset: Effect of adding chains on $I(Q)$ at $\rho_{s} \sigma_{s}^{3}=0.367$ for $\rho_{c} \sigma_{s}^{3}=0$ (circles) and $\rho_{c} \sigma_{s}^{3}=8.6$ (squares). Symbols: experiment; lines: theory.

fraction $\quad \phi^{*}=\left(f \bar{M}_{w} / \bar{d} N_{A}\right) /\left(4 \pi R_{m}^{3} / 3\right)=3 \% \quad\left(\rho_{s} \sigma_{s}^{3}=\right.$ $0.220)$, with $\bar{M}_{w}$ and $\bar{d}$ the average molar mass and mass density, and $R_{m}=R_{\text {core }}^{(s)}+R_{\text {shell }}^{(s)}$ (see Table I). The precise value of $\sigma_{s}$ was determined by optimizing the agreement between theory and experiment for $S(Q)$ at $\phi^{*}$, resulting in $\sigma_{s}\left(\phi^{*}\right)=305 \AA$, in good agreement with previous, independent estimates $[9,11]$ and within the experimental error bars of about $6 \%$ for $R_{g}^{(s)}$. The agreement between experiment and theory is very convincing. Position, height and width of first and second order peak of liquidlike experimental structure factors are indeed nicely described by the theory. Only at very low $Q$ vectors some deviations occur, probably due to not-perfect contrast matching conditions. In addition, from $\phi=5 \%\left(\rho_{s} \sigma_{s}^{3}=0.367\right)$ on, the height of the first structure factor peak is overestimated by theory due to the expected shrinkage of the micellar corona following $\sigma_{s} \sim\left(\phi / \phi^{*}\right)^{-1 / 8}$ [23]. This effect is shown in Fig. 3 for higher concentrations well above $\phi^{*}$. We point out that this shrinkage does not affect $\xi$ since the linear polymer also shrinks, following the same scaling relation. Consequently, the shrinkage is taken into account by readjusting the corresponding concentrations in terms of $\rho_{s} \sigma_{s}^{3}$ and $\rho_{c} \sigma_{s}^{3}$. The resulting theoretical $I(Q)$ are shown as continuous lines in Fig. 3 and clearly improve the agreement with experimental data. Similar effects are achieved for concentrations even as high as $20 \%\left(\approx 7 \phi^{*}\right)$. Nevertheless, the description of experimental data slightly worsens with concentration, most probably due to three body forces, which become relevant for $\phi / \phi^{*} \gtrsim 5$ [28].

To strengthen our quantitative comparison between experiment and theory, we also consider the phase behavior of the system. Indeed, while $S(Q)$ is only slightly affected by varying $\xi$, the phase behavior can be sensitively altered by a small change in the same. The agreement in phase behavior will provide an additional consistency check to narrow down the values of $\sigma_{s}, \sigma_{c}$ and $\xi$ and to establish

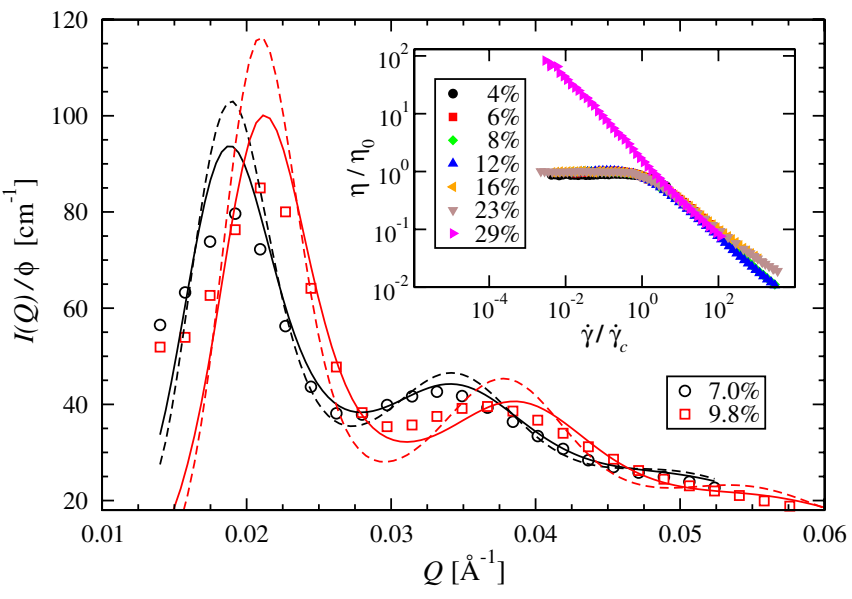

FIG. 3 (color online). Normalized SANS intensity for volume fractions well above $\phi^{*}$. Symbols correspond to experiments, dotted lines to theory with constant $\sigma$, and continuous lines to theory with decreasing $\sigma \sim\left(\phi / \phi^{*}\right)^{-1 / 8}$. Inset: Reduced viscosity vs reduced shear rate for selected volume fractions, with $\eta_{0}$ the zero shear viscosity and $\dot{\gamma}_{c}$ obtained from a Carreau-Fit [34].

the correctness of the adopted effective interactions. We investigated the rheological properties of the samples by means of both steady and oscillatory shear measurements. As the inset of Fig. 3 shows, most of viscosity curves display the Newtonian-plateau typical for a liquid and the usual shear-thinning at high shear rates. For larger concentration, namely $\phi=29 \%\left(\rho_{s} \sigma_{s}^{3}=0.887\right)$, we find a transition to a solid (glassy) state, clearly indicated by the absence of the Newtonian plateau. The formation of an arrested state was also verified for $\phi=33 \%\left(\rho_{s} \sigma_{s}^{3}=\right.$ 0.966 ) by tube inversion.

The theoretical phase diagram is shown in Fig. 4 for two different values of size ratios $\xi=0.27$ and $\xi=0.30$ in the $\left(\rho_{s}, \rho_{c}\right)$ plane. To account for the possible existence of a demixing region, we make use of the fact that the HNC fails to converge in the neighborhood of a spinodal line [25]. The convergence line (CL) in Fig. 4, can be considered an estimate of the phase separation region. Above the $\mathrm{CL}$, we expect the homogeneous mixture to become unstable with respect to demixing. Although it is possible to determine the coexistence curve from the HNC results [26], this is beyond the scope of this Letter, and therefore we keep the CL as an indicator of the location of phase separation. To estimate the stars' freezing boundary, we use the Hansen-Verlet (HV) criterion: to the right of the HV line, the main peak of $S(Q)$ exceeds 2.85, indicating crystallization [6]. Finally, an (ideal) glass transition line (GL) is shown, indicating vitrification [29]. This line was calculated by means of the (one-component) mode-coupling theory (MCT) [30], which uses as inputs only $S(Q)$ and $\rho_{s} \sigma_{s}^{3}$. This approach accounts very well for the location of the real vitrification line and that the addition of chains leads to glass melting at fixed $\rho_{s}$, as previously demonstrated $[12,13]$. 


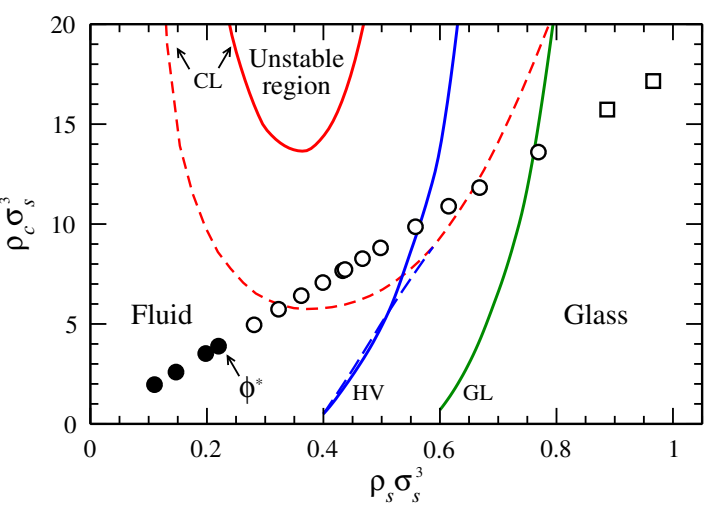

FIG. 4 (color online). Phase diagram: Symbols denote our experimental path (open symbols assuming shrinkage, open squares correspond to glassy samples). Lines (CL, HV, and GL, see text) are given by theory. Full and dashed lines correspond, respectively, to $\xi=0.3$ (the actual experimental one) and to $\xi=0.27$.

The investigated experimental points are also reported on this phase diagram. Given the constant ratio between starlike micelles and linear chains, our experimental path through the phase diagram is a straight line with slope $x_{n} /\left(1-x_{n}\right)$ with $x_{n}$ the number density fraction of the linear chains. For all samples under investigation, no phase separation was observed in agreement with the theoretical CL results for $\xi=0.30$. A crossing with the theoretical CL, which would happen for a smaller size ratio $\xi=0.27$, must be excluded. This result confirms that the actual experimental size ratio is close to $\xi=0.30$, as established by the $S(Q)$ analysis. At higher concentrations, our experimental path meets the HV line suggesting that a liquidsolid transition might take place. However a large number of studies on star polymer systems has shown that it is quite difficult to nucleate a crystal, while instead solutions at high concentrations undergo a disordered arrested state $[12,31,32]$. This was also the case for our starlike micelles in the absence of added chains at the same functionality as in the present study [6]. In the presence of chains, we also find that arrest is retarded to higher concentrations, in agreement with previous studies [12,31]. Indeed, for the most concentrated samples, the dynamical arrest to an amorphous solid (glass) agrees with the phase diagram of Fig. 4.

In summary, we have described structural and phase behavior of binary mixtures of ultrasoft colloids and linear polymers. By combining small-angle neutron scattering (SANS) and liquid state theory we offer robust experimental evidence to the accuracy of the coarse-graining procedure for developing effective interactions between the starlike micelles and the homopolymer chains, in a wide range of concentrated mixtures. Without any adjustable parameter we find quantitative agreement between experiments and theory for the influence of the added chains on the intermicelle structure and on their phase behavior. Our work provides a comprehensive characterization of soft binary mixtures in terms of effective potentials and is therefore a successful benchmark in the study of complex soft matter systems in general, opening the way for exploring experimentally the features of anomalous depletion predicted by theory [14], and for studying even more versatile soft-based systems, e.g., mixtures of micelles with block copolymers $[5,33]$.

We thank H. Cölfen for polymer characterization and C. Mayer for helpful discussions. M.C. thanks DAAD (Germany) and UAN-ICETEX-Colciencias (Colombia) for a PhD. Fellowship. This work was supported by the DFG-SFB TR6 and by the ITN-234810-COMPLOIDS.

[1] W. C. K. Poon, J. Phys. Condens. Matter 14, R859 (2002).

[2] S. Asakura and F. Oosawa, J. Polym. Sci. 33, 183 (1958).

[3] K. N. Pham et al., Science 296, 104 (2002).

[4] P. J. Lu et al., Nature (London) 453, 499 (2008).

[5] T. Lodge et al., Phys. Rev. Lett. 92, 145501 (2004).

[6] M. Laurati et al., Phys. Rev. Lett. 94, 195504 (2005).

[7] J. Mattson et al., Nature (London) 462, 83 (2009).

[8] F. Scheffold et al., Phys. Rev. Lett. 104, 128304 (2010).

[9] C. N. Likos et al., Phys. Rev. Lett. 80, 4450 (1998).

[10] M. Watzlawek, C. N. Likos, and H. Lowen, Phys. Rev. Lett. 82, 5289 (1999).

[11] E. Stiakakis et al., Phys. Rev. Lett. 89, 208302 (2002).

[12] E. Stiakakis et al., Europhys. Lett. 72, 664 (2005).

[13] E. Zaccarelli et al., Phys. Rev. Lett. 95, 268301 (2005).

[14] M. Camargo and C. N. Likos, Phys. Rev. Lett. 104, 078301 (2010).

[15] C. Mayer et al., Nature Mater. 7, 780 (2008).

[16] C. Mayer and C. N. Likos, Macromolecules 40, 1196 (2007).

[17] A. Wilk et al., Eur. Phys. J. E 32, 127 (2010).

[18] R. Lund et al., Phys. Rev. Lett. 96, 068302 (2006).

[19] J. S. Pedersen, J. Chem. Phys. 114, 2839 (2001).

[20] A. Poppe et al., Macromolecules 30, 7462 (1997).

[21] A. Jusufi et al., J. Phys. Condens. Matter 13, 6177 (2001).

[22] R. L. C. Vink et al., Phys. Rev. E 72, 030401(R) (2005).

[23] M. Daoud and J. P. Cotton, J. Phys. (Paris) 43, 531 (1982).

[24] We evaluated the density profiles $\varrho_{i}(r)$ following [16]. We employed the fitting parameter $\kappa \sigma_{i}=1.90$, which results in a better fit of the effective interaction (3) compared to simulation results as functionality increases $(f \sim 100)$.

[25] J.P. Hansen and I. R. McDonald, Theory of simple liquids (Academic Press, London, 2006).

[26] A. J. Archer et al., J. Phys. Condens. Matter 14, 12031 (2002).

[27] J. S. Pedersen, Adv. Colloid Interface Sci. 70, 171 (1997).

[28] C. von Ferber et al., Eur. Phys. J. E 2, 311 (2000).

[29] G. Foffi et al., Phys. Rev. Lett. 90, 238301 (2003).

[30] W. Götze, in Liquids, Freezing and Glass Transition, edited by J. P. Hansen, D. Levesque, and J. Zinn-Justin (North-Holland, Amsterdam, 1991).

[31] B. Loppinet et al., Macromolecules 34, 8216 (2001).

[32] E. Stiakakis et al., Phys. Rev. E 81, 020402(R) (2010).

[33] S. Abbas and T. Lodge, Phys. Rev. Lett. 99, 137802 (2007).

[34] P. J. Carreau, Trans. Soc. Rheol. 16, 99 (1972). 